\title{
Report of a successful ongoing pregnancy as a result of IMSI with assisted oocyte activation
}

\author{
Bogdan Doroftei ${ }^{1,2,3}$, Mihaela Zlei $^{3,4,5^{*}}$, Gabriela Simionescu ${ }^{2,3}$, Radu Maftei ${ }^{2,3}$, Simona Cumpata ${ }^{3}$ \\ and Geraldine Emerson ${ }^{3}$
}

\begin{abstract}
We report a successful ongoing pregnancy obtained in a case of total globozoospermia after intracytoplasmic morphologically selected sperm injection (IMSI) with oocyte activation. The first semen analysis on investigation showed partial globozoospermia. However, under high magnification assessment at oocyte retrieval only round headed sperm were observed. Considering the high risk of a complete failure to fertilize from IMSI the couple gave written informed consent to the use of oocyte activation media post IMSI. One embryo fertilized, developed to a hatching blastocyst and was transferred resulting in an ongoing pregnancy. This successful outcome shows the use of IMSI is useful in the evaluation of total globozooozpermia and therefore aids in the justification of the use of oocyte activation media.
\end{abstract}

Keywords: Globozoospermia, Artificial Oocyte Activation, IMSI

\section{Background}

In globozoospermia the main morphological defect is characterized by the absent or severely malformed acrosome. The pathogenesis occurs during spermiogenesis and probably originates in the acrosomic vesicle fusion impairment and cytoskeleton disorders, although precise mechanisms remain to be determined [1]. Total (100\% round headed spermatozoa) or partial (less than $100 \%$ ) globozoospermia have been described [2,3].

The introduction of intracytoplasmic sperm injection (ICSI) and then morphologically selected sperm injection (IMSI) lead the way for males with severe globozoospermia to have the ability to father their own children. However, rates of fertilization remained poor for this cohort of males and it quickly became evident that round headed sperm did not have the ability to trigger oocyte activation [4-6]. In 1997, the first reports of assisted oocyte activation (AOA) and improved fertilization could be achieved by applying calcium ionophore in such cases [7]. Here we will report on a successful ongoing pregnancy after IMSI with oocyte activation.

\footnotetext{
* Correspondence: mihaelazlei@gmail.com

${ }^{3}$ Origyn Fertility Center, lasi, Romania

${ }^{4}$ Regional Institute of Oncology, lasi, Romania

Full list of author information is available at the end of the article
}

\section{Case presentation}

A 32 years old couple attended for fertility consultation reporting a history of 5 years trying for pregnancy with no success. On investigation of the female the fertility workup included: check ovarian reserve by antimullerian hormone $3.72 \mathrm{ng} / \mathrm{mL}$ and antral follicle count 18 follicles on both ovaries, ultrasound examination of uterus and office diagnostic hysteroscopy. All showed no abnormalities. Semen analysis on the male showed a volume of $5.6 \mathrm{~mL}$, concentration of 23 million/ $\mathrm{mL}, 44 \%$ motile of which only $5 \%$ were progressively motile. Sperm morphology was reported as $2 \%$ normal with evidence of acrosome abnormalities (strict criteria, Figure 1). DNA fragmentation index was $10.6 \%$ and high DNA stainability was $14.9 \%$. Both were found by flow-cytometry method.

Male factor infertility was diagnosed and the couple counseled for IMSI. Informed consent was obtained. No history was known regarding other male family members fertility.

The female patient was treated with antagonist protocol using ganirelix (Orgalutran - MSD) and the administration of $150 \mathrm{IU}$ rFSH (Puregon - MSD) daily for 9 days. Oocyte retrieval was undertaken 36 hours post hCG priming 10,000 IU (Pregnyl - MSD). A total of 7 oocytes were collected of which 5 were mature and 


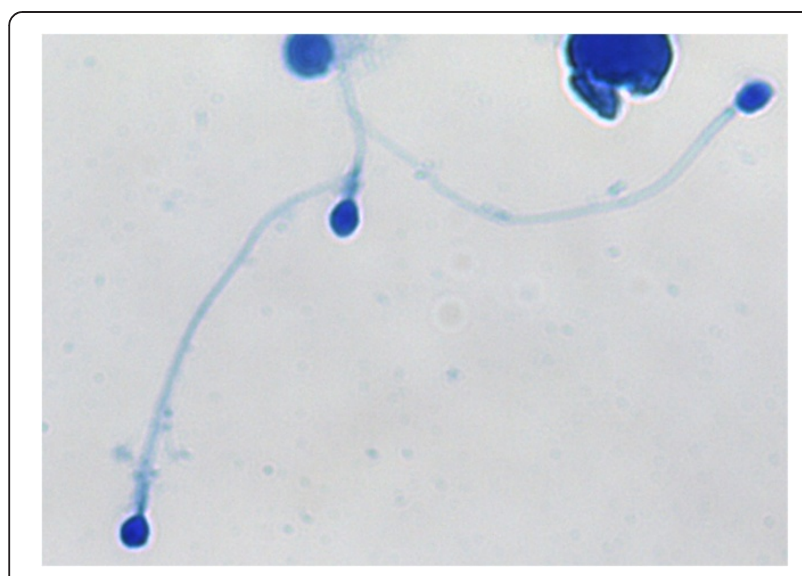

Figure 1 Microscopy image of sperm morphologic assessment.The staining was performed with SpermBlue (Microptic, Spain). Magification with the oil immersion, 100 x objective (Leica GX, L3200, Leica Application Capture Software).

suitable for injection. Semen analysis on the day of oocyte retrieval on high magnification using an inverted microscope equipped with Nomarski differential interference contrast optics (Leica AM 6000) showed no normal morphology only total round heads (globozoospermia). Due to the concern of a total failure to fertilize the couple were informed that IMSI would be the method of choice to continue to observe the sperm sample, trying to identify any sperm with a partial or small acrosome to avoid the use of oocyte activation media. However none were observed and post IMSI the use of oocyte activation media was undertaken. The couple were fully informed of the limited data available on the use of calcium ionophore with regard to the long-term health of resultant offspring. Written consent was obtained to carry out this procedure. The characteristics of the sperm used for insemination showed total round head nucleus with no acrosome present, however all were motile.

Following IMSI the 5 oocytes were placed into oocyte activation media (GM508, Cult Active, GYNEMED) for 15 minutes at 37 degrees 5\% 02 and 6\% CO2. The oocytes were then washed free of calcium ionophore through 8 drops of culture media. The oocytes were cultured over night in CSCM media (Irvine Scientific, Santa Ana, CA) in the embryoscope time lapse incubator (FertilitechUnisence Denmark). Sixteen hours post injection only one oocyte showed signs of fertilization and developed normally (Figure 2; an additional movie file shows this in details, see Additional file 1).

The embryo was cultured to day 5 uninterrupted in the embryoscope (Unisense FertiliTech, Aarhus, Danemarca) and a blastocyst grade 6AA [8] was transferred under ultrasound guidance. BhCG level on day 12 post embryo transfer was $468.9 \mathrm{mUI} / \mathrm{mL}$, 2 days later BhCG level was $916.1 \mathrm{mUI} / \mathrm{mL}$. Ultrasound evaluation at 12 weeks and 5 days of gestation (Figure 3) showed Fetal Heart Beat $149 \mathrm{bpm}$, CRL (crown-rump length $63 \mathrm{~mm}$, nuchal transluency $-1.6 \mathrm{~mm}$ and intracranianal transluency $2.1 \mathrm{~mm}$, ductus venosus dopller without notch, nasal bone present and tricuspid doppler in normal range. First trimester biochemical and ultrasound screening reveal low risk for trisomy.

As yet, it is unclear whether patients whose ejaculate contains both $100 \%$ globozoospermicornormal and

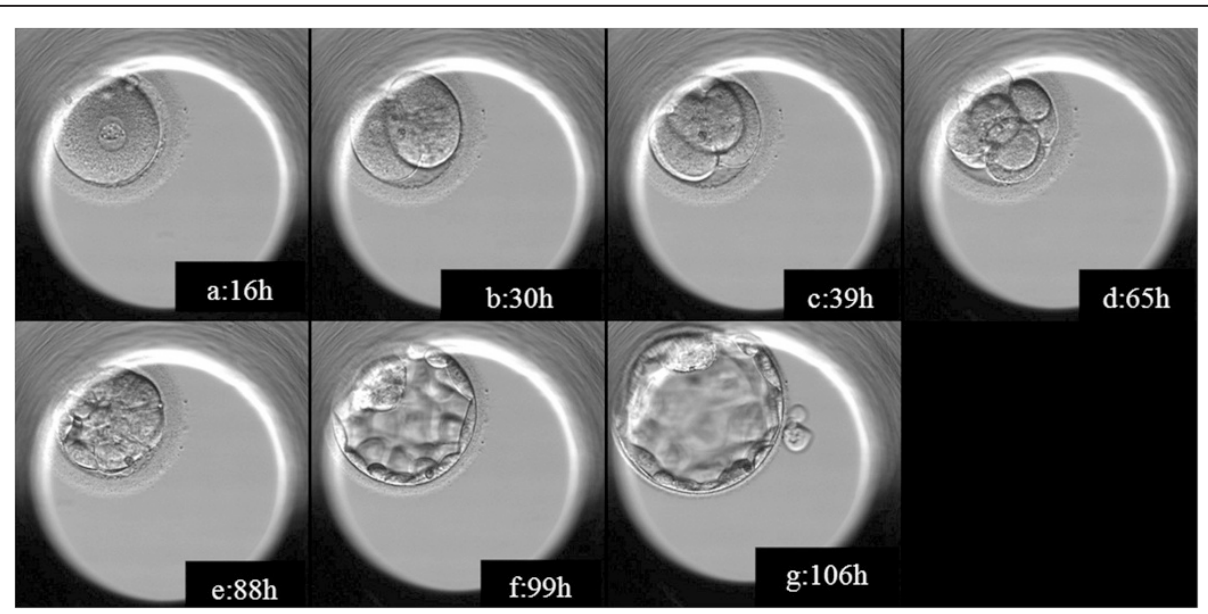

Figure 2 Different stages of embryo development captured with a Time Lapse System. The images were captured (Embrioscope, FertilitechUnisence Denmark, Embryo-Viewer Software) at different stages of the embryo's development post injection: a - 2 pro-nucleai, 16 hours, $\mathbf{b}-2$ cells (30 hours), $\mathbf{c}-4$ cells (39 hours), $\mathbf{d}-8$ cells ( 65 hours), e - start of blastocyst ( 88 hours), $\mathbf{f}$ - expanded blastocyst ( 99 hour), $\mathbf{g}-$ hatching blastocyst (106 hours). 


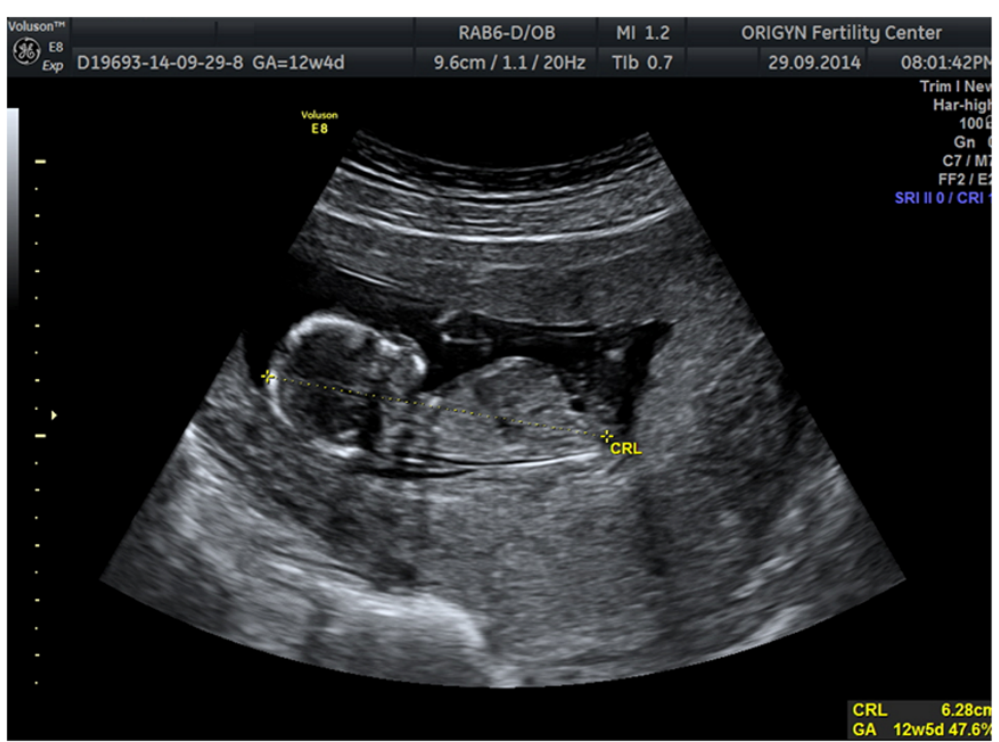

Figure 3 Ultrasound image of the fetus at 12 weeks and 2 days of gestation.

globozoospermic cells (partial globozoospermia) suffer from a variation of the same syndrome [1] and still remains to be elucidated. Yoon et al. [9] reported absence of $\mathrm{PLCz}$ in both the normal and round-headed sperm of a partial globozoospermic patient, in line with the inability of partial globozoospermic sperm cell types to activate mouse oocytes as reported by Heindryckx et al. [10]. Numerous reports studying familial cases have suggested that globozoospermia is a genetic syndrome [11-15]. However, the specific mode of inheritance remains unclear, although recently it was reported that a mutation in the SPATA16 gene appears to be associated with certain types of globozoospermia in men [1].

\section{Conclusions}

To our knowledge this is the first reported ongoing pregnancy after IMSI using globozoospermic spermatozoa with AOA in Romania. This report shows the use of high magnification tools such as IMSI in the assessment of sperm morphology can aid in the reduction of complete failure to fertilize in cases of suspected globozoospermia with the introduction of AOA when necessary.

\section{Consent}

Written informed consent was obtained from the patient for publication of this Case report and any accompanying images. A copy of the written consent is available for review by the Editor-in-Chief of this journal.

\section{Additional file}

Below is the link to the electronic supplementary material.

\section{Additional file 1: Morphokinetic illustration of the embryo} development, captured with a Time Lapse System. The images were captured using the Embrioscope from Fertilitech-Unisence Denmark; the software used was Embryo-Viewer Software. Images were capture every 15 minutes, using 4 focal planes.

\section{Abbreviations}

ICSI: Intracytoplasmic sperm injection; IMSI: Morphologically selected sperm injection; AOA: Assisted oocyte activation.

\section{Competing interests}

The authors declare that they have no competing interests.

\section{Authors' contributions}

All authors read and approved the final manuscript and agreed to be accountable for all aspects of the work in ensuring that questions related to the accuracy or integrity of any part of the work are appropriately investigated and resolved. BD conceived of the treatment scheme, and participated in its design and coordination, helped to draft the manuscript, and have given final approval of the version to be published, has been involved in patient's treatment, follow-up, ultrasound evaluation and has made substantial contributions to interpretation of data. MZ has been involved in drafting the manuscript, revising it critically for important intellectual content, and performed the DNA fragmentation analysis by flow-cytometry. GS has been involved in patient's treatment, follow-up, ultrasound evaluation and has made substantial contributions to interpretation of data and manuscript revision. RM has been involved in patient's treatment, follow-up, ultrasound evaluation and has made substantial contributions to interpretation of data. SC has been involved in revising the manuscript critically for important intellectual content, and performed the sperm analysis, culture and assessment of the embryos. GE has been involved in drafting the manuscript, revising it critically for important intellectual content, has made substantial contributions to conception and design of the manuscript and interpretation of data, and performed the IMSI procedure, culture and assessment of the embryos. 


\section{Authors' information}

BD: MD, PhD, Affiliations: University of Medicine and Pharmacy, Obstetrics and Gynecology Department, lasi; Teaching Hospital Obgyn "Cuza Voda", lasi, Romania; Origyn Fertility Center, lasi, Romania

MZ: PhD;Affiliations: Regional Institute of Oncology, lasi, Romania; Origyn Fertility Center, lasi, Romania

GS: MD; Affiliations:Teaching Hospital Obgyn "Cuza Voda", lasi, Romania; Origyn Fertility Center, lasi, Romania

RM: MD; Affiliations: Teaching Hospital Obgyn "Cuza Voda", lasi, Romania; Origyn Fertility Center, lasi, Romania

SC: Affiliation: Origyn Fertility Center, lasi, Romania

GE: PhD;Affiliation: Origyn Fertility Center, lasi, Romania.

\section{Acknowledgements}

The current survey was supported by National Authority of Scientific Research of Romania (POSCCE Grant 1357 "Infertility a tree pieces puzzle: couple investigation, infertility diagnostic, possible therapy").

\section{Author details}

'Obstetrics and Gynecology Department, University of Medicine and Pharmacy, lasi, Romania. 'Teaching Hospital Obgyn "Cuza Voda", Iasi, Romania. ${ }^{3}$ Origyn Fertility Center, lasi, Romania. ${ }^{4}$ Regional Institute of Oncology, lasi, Romania. ${ }^{5}$ Laboratory of Molecular Biology, Regional Institute of Oncology, Romania, 2-4 Berthelot Street, postal code 700483 lasi, Romania.

Received: 20 November 2014 Accepted: 29 April 2015

Published online: 03 May 2015

\section{References}

1. Dam AH, Koscinski I, Kremer JA, Moutou C, Jaeger AS, Oudakker AR, et al. Homozygous mutation in SPATA16 is associated with male infertility in human globozoospermia. Am J Hum Genet. 2007;81(4):813-20.

2. Holstein AF, Schirren CG, Schirren C, Mauss J. Round headed spermatozoa: a cause of male infertility. Dtsch Med Wochenschr. 1973;98(2):61-2.

3. Dam AH, Ramos L, Dijkman HB, Woestenenk R, Robben H, van den Hoven L, et al. Morphology of partial globozoospermia. J Androl. 2011;32(2):199-206.

4. Saunders CM, Larman MG, Parrington J, Cox LJ, Royse J, Blayney LM, et al. PLC zeta: a sperm-specific trigger of $\mathrm{Ca}(2+)$ oscillations in eggs and embryo development. Development. 2002;129(15):3533-44.

5. Grasa P, Coward K, Young C, Parrington J. The pattern of localization of the putative oocyte activation factor, phospholipase Czeta, in uncapacitated, capacitated, and ionophore-treated human spermatozoa. Hum Reprod. 2008;23(11):2513-22

6. Ikawa M, Inoue N, Benham AM, Okabe M. Fertilization: a sperm's journey to and interaction with the oocyte. J Clin Invest. 2010;120(4):984-94.

7. Rybouchkin AV, Van der Straeten F, Quatacker J, De Sutter P, Dhont M. Fertilization and pregnancy after assisted oocyte activation and intracytoplasmic sperm injection in a case of round-headed sperm associated with deficient oocyte activation capacity. Fertil Steril. 1997;68(6):1144-7.

8. Alpha Scientists in Reproductive Medicine and ESHRE Special Interest Group of Embryology. The Istanbul consensus workshop on embryo assessment: proceedings of an expert meeting. Hum Reprod. 2011;26(6):1270-83.

9. Yoon SY, Jellerette T, Salicioni AM, Lee HC, Yoo MS, Coward K, et al. Human sperm devoid of PLC, zeta 1 fail to induce $\mathrm{Ca}(2+)$ release and are unable to initiate the first step of embryo development. J Clin Invest. 2008;118(11):3671-81.

10. Heindryckx B, De Gheselle S, Gerris J, Dhont M, De Sutter P. Efficiency of assisted oocyte activation as a solution for failed intracytoplasmic sperm injection. Reprod Biomed Online. 2008;17(5):662-8.

11. Kullander S, Rausing A. On round-headed human spermatozoa. Int J Fertil. 1975;20:33-40.

12. Flörke-Gerloff S, Töpfer-Petersen E, Müller-Esterl W, Mansouri A, Schatz R, Schirren C, et al. Biochemical and genetic investigation of round-headed spermatozoa in infertile men including two brothers and their father. Andrologia. 1984;16(3):187-202

13. Dale B, laccarino M, Fortunato A, Gragnaniello G, Kyozuka K, Tosti E. A morphological and functional study of fusibility in round-headed spermatozoa in the human. Fertil Steril. 1994;61(2):336-40.
14. Kilani Z, Ismail R, Ghunaim S, Mohamed H, Hughes D, Brewis I, et al. Evaluation and treatment of familial globozoospermia in five brothers. Fertil Steril. 2004;82(5):1436-9.

15. Heindryckx B, Van der Elst J, De Sutter P, Dhont M. Treatment option for sperm- or oocyte-related fertilization failure: assisted oocyte activation following diagnostic heterologous ICSI. Hum Reprod. 2005;20(8):2237-41.

\section{Submit your next manuscript to BioMed Central and take full advantage of:}

- Convenient online submission

- Thorough peer review

- No space constraints or color figure charges

- Immediate publication on acceptance

- Inclusion in PubMed, CAS, Scopus and Google Scholar

- Research which is freely available for redistribution

Submit your manuscript at www.biomedcentral.com/submit 